\title{
MAPPING CYLINDER NEIGHBORHOODS IN THE PLANE
}

\author{
BEVERLY BRECHNER AND MORTON BROWN
}

\begin{abstract}
We characterize those compact subsets of the plane which have mapping cylinder neighborhoods, describe the neighborhood closures, and show that such neighborhood closures are topologically unique. The proofs employ the notion of prime ends. We also show that if $U$ is a mapping cylinder neighborhood of a pointlike continuum in $S^{3}$, then $\bar{U}$ is a 3 -cell.
\end{abstract}

1. Introduction. What are the possible spines of a two-disk $D^{2}$ ? That is, which compact subsets $X$ of the plane $R^{2}$ have mapping cylinder neighborhood closures (see below) homeomorphic to $D^{2}$ ? The question was raised to one of the authors by Herman Gluck. In this note, we show (Theorem 2.1) that it is necessary and sufficient that $X$ be a nonseparating Peano continuum. The answer, although not surprising, seems not to be in the literature. We provide a short proof based upon Carathéodory's Theory of Prime Ends [7]. See [2, Section 2] for a brief description, including examples. Also see $[13,16]$.

In addition, in Theorem 2.4, we show that mapping cylinder neighborhoods in the plane, when they exist, are unique. In particular, their closures are unique.

We thank the referee for raising a question which led to this latter result.

Notation And Definitions. A double arrow $(\rightarrow)$ means that the function is onto, and Int, and Bd mean interior, and boundary respectively.

We use the following definitions (compare [11]): The mapping cylinder $M_{f}$ of a map $f$ of a space $X$ onto a space $Y$ is the disjoint union $(X \times[0,1]) \cup Y$ with each $(x, 1)$ identified to $f(x) \in Y$. By identifying each $x \in X$ with $(x, 0)$ in $M_{f}$, we may consider $X, Y$ as closed subsets of $M_{f}$. A mapping cylinder neighborhood of a closed subset $X$ of a topological space $Z$ is a pair $((U, \operatorname{Bd} U, X), f: \operatorname{Bd} U \rightarrow \operatorname{Bd} X)$ where $U$ is an open set containing $X, f$ is continuous, and such that there exists a homeomorphism $h: M_{f} \rightarrow[\bar{U}-$ Int $X]$ with $h \mid(\operatorname{Bd} U \cup \operatorname{Bd} X)=1$. We will call the open set $U$ an open mapping cylinder neighborhood while $\bar{U}$ is called a closed mapping cylinder neighborhood. But we keep in mind all of the above structure, in both cases.

REMARKS. (1) From the above, we see that if $U$ is an open mapping cylinder neighborhood of $X$ with respect to the map $f$, then $(\bar{U}-\operatorname{Int} X)$ may be thought of, as the mapping cylinder $M_{f}$.

(2) Note that if $M_{f}$ is the mapping cylinder associated with the open mapping cylinder neighborhood $U$ of $X$ in $Z$, then the base of $M_{f}$ (identified with $\mathrm{Bd} X$ by a

Received by the editors December 30, 1978 and, in revised form, June 1, 1981.

1980 Mathematics Subject Classification. Primary 54C10, 54C15, 54C20, 54F65, 57A05.

Key words and phrases. Mapping cylinder neighborhood, prime ends, plane homeomorphisms.

() 1982 American Mathematical Society 0002-9939/81/0000-1039/\$02.00 
homeomorphism) is a strong deformation retract of $M_{f}$. Thus $X$ is a strong deformation retract of $\bar{U}$.

\section{Main theorems.}

2.1. TheOREM. A compact subset $X$ of the plane has an open mapping cylinder neighborhood whose closure is a disk iff $X$ is a nonseparating Peano continuum iff $X$ is an absolute retract. ${ }^{1}$

Proof. Assume that $X$ has an open mapping cylinder neighborhood whose closure is a disk. Then $X$ is a (deformation) retract of a disk and therefore $X$ is an absolute retract. Thus $X$ is a Peano continuum [8, p. 232] and, having no essential maps onto the circle, cannot separate the plane [12, p. 100].

Conversely, assume that $X$ is a nonseparating Peano continuum. We think of $X$ as embedded in the 2-sphere $S^{2}$, and we get a $C$-map $\phi$ from $S^{2}-X$ onto the interior of the unit disk [ 2 or 16]. Since $X$ is locally connected, each prime end of $S^{2}-X$ is of the first kind [1]. Hence, by [13, p. 67], $\phi^{-1}$ can be extended to a map $F$ : $D^{2} \rightarrow\left(S^{2}-\right.$ Int $\left.X\right)$ such that $F\left(\operatorname{Bd} D^{2}\right)=\operatorname{Bd} X$. Then

$$
U=X \cup F\left(D^{2}-\left\{x \in R^{2} \mid\|x\| \leqslant \frac{1}{2}\right\}\right)
$$

is the required open mapping cylinder neighborhood of $X$. Note that since $F$ is a homeomorphism on Int $D^{2}$, and $T=\left\{x \in R^{2} \mid\|x\|=\frac{1}{2}\right\}$ is a compact subset of $D^{2}$ missing $\mathrm{Bd} D^{2}, F(T)=\operatorname{Bd} U$, and $\mathrm{Bd} U$ is a simple closed curve. Thus $\bar{U}$ is a disk.

2.2. Lemma. For $n \geqslant 1$, statements (i) and (ii) are equivalent. For $n=2$, (i), (ii) and (iii) are equivalent:

(i) $A$ subset $C$ of $S^{n}$ is compact and pointlike (i.e., $S^{n}-C \approx E^{n}$ ),

(ii) $C$ is cellular in $S^{n}$,

(iii) $C$ is a nonseparating continuum in $S^{n}$.

Proof. (i) $\Leftrightarrow$ (ii) is due to M. Brown [4]. That (i) $\Leftrightarrow$ (iii) for $n=2$, can be found in [14].

2.3. Lemma. Let $n=2,3$. If $Y$ is a topological space and $C \circ Y$, the cone on $Y$, is locally Euclidean of dimension $n$, then $Y$ is homeomorphic to the $(n-1)$-sphere $S^{n-1}$.

Proof. Since the cone on $Y$ is locally Euclidean at the cone point, and Euclidean space is locally compact, it follows that $Y$ is compact. According to Theorem 4 of [15], the suspension of $Y, S^{\circ} \circ Y$, is homeomorphic to $S^{n}$. Removing the suspension points, we see that $Y \times R^{1}$ is homeomorphic to $S^{n-1} \times R^{1}$ and hence $Y$ is locally connected.

Suppose now that $n=2$. Then no point separates $Y$. For if $p$ separates $Y$, then the $\operatorname{arc} S^{\circ} \circ\{p\}$ separates $S^{\circ} \circ Y \approx S^{2}$. On the other hand, if $\{p, q\}$ is a pair of points in $Y$ then $S^{\circ} \circ\{p, q\}$ is a l-sphere in $S^{2}$ and separates $S^{2}$. Removing the suspension points we see that $\{p, q\} \times R^{1}$ separates $Y \times R^{1}$ so that $\{p, q\}$ separates $Y$. Thus $Y$

\footnotetext{
${ }^{1}$ That absolute retracts in the plane are the same collection as nonseparating Peano continua is well known (see V. 13.1 of [3]). Incidentally, this theorem provides a new proof.
} 
is a nondegenerate, locally connected metric continuum separated by no point and by every pair of points, and is therefore a simple closed curve. If $n=3$, the same kind of argument shows that $Y$ is separated by no arc and by every simple closed curve (and it must contain a simple closed curve). Thus, by the Kline Sphere Characterization or Theorem VI.5.1 of [17], $Y$ must be a 2-sphere.

REMARK. If $n \geqslant 4$, then Lemma 2.3 is false (see the introduction in [5]), even for $Y$ a finite simplicial complex when $n=4$ [9].

2.4. TheOREM. Let $X$ be a pointlike continuum in $S^{n}(n=2,3)$ with a mapping cylinder neighborhood $U$. Then $\bar{U}$ is an $n$-cell.

Proof. Let $f: \operatorname{Bd} U \rightarrow \operatorname{Bd} X$ define the mapping cylinder neighborhood $U$ of $X$. Since $X$ is cellular in $S^{n}$, by Theorem 1 of [4], there is a homeomorphism $(\bar{U}$, $\operatorname{Bd} U) \approx(\bar{U} / X, \operatorname{Bd} U)$. On the other hand,

$$
\begin{aligned}
(\bar{U} / X, \operatorname{Bd} U) & \approx((\bar{U}-\operatorname{Int} X) / \operatorname{Bd} X, \operatorname{Bd} U) \\
& \approx\left(M_{f} / \operatorname{Bd} X, \operatorname{Bd} U\right) \approx(C \circ(\operatorname{Bd} U), \operatorname{Bd} U) .
\end{aligned}
$$

Thus $(\bar{U}, \operatorname{Bd} U) \approx(C \circ(\operatorname{Bd} U), \operatorname{Bd} U)$ with the cone point $c$ corresponding to a point of $\bar{U}-\mathrm{Bd} U=U$. Thus $C \circ(\mathrm{Bd} U)$ is locally Euclidean at the point $c$. By Lemma 2.3, $\operatorname{Bd} U$ is an $(n-1)$-sphere. Since $\operatorname{Bd} U$ is collared in $C \circ(\operatorname{Bd} U), \bar{U}$ is an $n$-cell, by [4].

2.5. Corollary. A compact subset of $E^{2}\left(S^{2}\right)$ has an open mapping cylinder neighborhood iff it has only a finite number of components, only a finite number of complementary domains, and each component is a Peano continuum. Further, any two open mapping cylinder neighborhoods have homeomorphic closures-a 2-manifold which is a disk with finitely many holes.

\section{REFERENCES}

1. B. Brechner and R. D. Mauldin, Positively regular homeomorphisms of Euclidean spaces, Topology Appl. 11 (1980), 231-242.

2. B. Brechner, On stable homeomorphisms and imbeddings of the pseudo arc, Illinois J. Math. 22 (1978), 630-661.

3. K. Borsuk, Theory of retracts, PWN, Warsaw, 1967.

4. M. Brown, A proof of the generalized Schoenflies theorem, Bull. Amer. Math. Soc. 66 (1960), 74-76.

5. ___ Wild cells and spheres in higher dimensions, Michigan Math. J. 14 (1967), 219-224.

6. The monotone union of open n-cells is an open n-cell, Proc. Amer. Math. Soc. 12 (1961), $812-814$.

7. C. Carathéodory, Über de Begrenzung einfach zuzammenhängender Gebiete, Math. Ann. 73 (1913), 323-370.

8. C. Christenson and W. Voxman, Aspects of topology, Dekker, New York and Basel, 1975.

9. R. D. Edwards, The double suspension of a certain homology 3-sphere is $S^{5}$, Notices Amer. Math. Soc. 22 (1975), A 334. Abstract \#75T-533.

10. The topology of manifolds and cell-like maps, preprint dated 5/79.

11. K. Kwun and F. Raymond, Mapping cylinder neighborhoods, Michigan Math. J. 10 (1963), 353-357.

12. W. Hurewicz and H. Wallman, Dimension theory, Princeton Univ. Press, Princeton, N. J., 1948.

13. A. I. Markushevich, Theory of functions of a complex variable, vol. III, rev. English ed., Prentice-Hall, Englewood Cliffs, N. J., 1965.

14. R. L. Moore, Concerning upper semi-continuous collections of continua, Trans. Amer. Math. Soc. 27 (1925), 416-428. 
15. R. H. Rosen, Stellar neighborhoods in polyhedral manifolds, Proc. Amer. Math. Soc. 14 (1963), 401-406.

16. H. D. Ursell and L. C. Young, Remarks on the theory of prime ends, Mem. Amer. Math. Soc., No. 3, 1951.

17. G. T. Whyburn, Analytic topology, Amer. Math. Soc. Colloq. Publ., vol. 28, Amer. Math. Soc., Providence, R. I., 1942.

Department of Mathematics, University of Florida, Gainesville, Florida 32611

Department of Mathematics, University of Michigan, ANn Arbor, Michigan 48109 